\section{Men who have sex with men in China have relatively low numbers of sexual partners}

\author{
Lei Zhang, Eric Pui Fung Chow, \\ David P. Wilson
}

The Kirby Institute for infection and immunity in society, University of New South Wales, Sydney, Australia

\begin{abstract}
HIV prevalence is increasing rapidly among men who have sex with men (MSM) in China and potentially associated with the number of male sexual partners that each man has on average. This study estimates the distribution of the number of male sexual partners among Chinese MSM through a comprehensive review of English and Chinese published literature. The overall median number of male sexual partners of Chinese MSM in the past 6 months China was estimated to be $1.5(95 \% \mathrm{CI}$, 1.1-1.8) and 3.8 (95\% CI 1.5-6.9) sexual partners in the past 6 -month and 12 -month periods respectively. An estimated $31 \%$ of sexual partners of MSM in China are regular partners, $54 \%$ are casual partners, and $16 \%$ are commercial partners. The reported numbers of sexual partners has not changed over time during the past decade. The numbers of male sexual partners reported by Chinese MSM is consistently lower than other settings and may not be sufficient to explain the recent rapid increase in HIV prevalence.
\end{abstract}

\section{Introduction}

There are an estimated 5-10 million men who have sex men (MSM) in China. ${ }^{1}$ MSM in China are a population group at increasing risk for HIV infection. A recent national report revealed that the proportion of new HIV annual infections due to homosexual exposure has increased from $12-33 \%$ over the past 3 years, 2007-2009. ${ }^{2}$ Estimated HIV prevalence among MSM in China has increased from 1.4-5.3\% during 2001-2009. ${ }^{3}$

HIV transmission is strongly associated with levels of engaging in risk behaviors. ${ }^{4}$ Behavioral surveillance of risk factors associated with HIV is valuable for identifying targets for public health efforts. There are limited systems in place for collecting routine behavioral surveillance data for at-risk populations in China, particularly for MSM. Currently China has 1029 national HIV sentinel surveillance sites, ${ }^{5}$ but only 56 specifically targeted
MSM. ${ }^{6}$ There is considerable diversity in the risk behaviors engaged in by Chinese MSM, including their condom usage, number of sexual partners and frequency of sexual acts. Thus, not only is it important to estimate average levels of risk but also to obtain knowledge of the distribution of behaviors across the population. A comprehensive literature review over all available published studies is a valuable way of collating information to estimate the overall level of risk behaviours in the population. In this study we estimate the distribution of numbers of male sexual partners among Chinese MSM through a literature review of both English and Chinese published journal articles during 2000-2010.

\section{Materials and Methods}

We conducted a review of published articles in the following Chinese and English electronic databases: PubMed, Chinese Scientific Journals Fulltext Database, China National Knowledge Infrastructure (CNKI) and Wanfang Data (Figure 1). Keywords used in the search included (HIV or AIDS, sexual behavior or risk behavior) and (homosexual or gay or bisexual or Tongzhi, Chinese term referring to homosexual men) or (men who have sex with men or MSM) and China. Studies published during 2000-2010 were included if they reported numbers of male sexual partners in the last six or twelve months with at least 3 partner sub-categories and information about the study (year, location and sampling size). Data from all studies were collated and categorised according to types of sexual partnerships where possible (regular, non-commercial casual, commercial, and unspecified). The literature search was performed by two investigators (EPFC, LZ) independently and the search results were then compared. The two investigators discussed to resolve any differences in the research results and undetermined publications were decided by the third investigator (DPW).

Lognormal distributions represent probability distributions of a random variable whose logarithmic values are normal distributed and have been accepted as a good approximation to the distribution of sexual partners of human populations. ${ }^{7}$ We hence fitted cumulative lognormal distributions to the cumulative numbers of sexual partners from collated studies. 95\% confidence intervals (CI) were generated by 100 repeated bootstraps on the fitted curves. We investigate the temporal trend of number of sexual partners of MSM in the past 10 years using the Spearman non-parametric correlation test $(\mathrm{P}<0.05$ indicates statistically significant trend).
Correspondence: Lei Zhang, CFI Building, Corner West and Boundary Streets, Darlinghurst, Sydney, NSW 2010, Australia.

E-mail: lzhang@nchecr.unsw.edu.au

Tel. +61.2.9385.0900 - Fax. +61.2.9385.0920.

Key words: HIV, men who have sex with men, review, China.

Acknowledgements: the authors acknowledge funding from the Australian Research Council and the University of New South Wales. The Kirby Institute for infection and immunity in society is funded by the Australian Government, Department of Health and Ageing. The views expressed in this publication do not necessarily represent the position of the Australian Government. The Kirby Institute for infection and immunity in society with the Faculty of Medicine at the University of New South Wales.

Conflict of interest: the authors report no conflicts of interest.

Received for publication: 29 April 2011.

Revision received: 3 Jume 2011.

Accepted for publication: 8 June 2011.

This work is licensed under a Creative Commons Attribution NonCommercial 3.0 License (CC BYNC 3.0).

(C) Copyright L. Zhang et al., 2011

Licensee PAGEPress, Italy

Infectious Disease Reports 2011; 3:e10

doi:10.4081/idr.2011.e10

\section{Results}

We identified 749 articles from four electronic databases (105 in PubMed, 194 in CQVIP, 224 in CNKI and 226 in Wanfang), we also identified 15 articles from reference lists of published articles. We removed 506 articles due to duplication records in other databases and unrelated title. The remaining 258 articles were screened and we further excluded 163 articles after screening of abstracts ( 86 were non peer-reviewed theses, 33 were news articles, 23 were conference abstract and 21 were review articles). The remaining 95 articles were eligible for full-text screening, we excluded 74 articles at this stage (3 were duplicated studies; 3 reported number of sexual partners in last three months; 12 contained less than three data points in the number of sexual partners' distribution and 56 did not report the number of sexual partners among MSM). Finally, there were 21 eligible articles ( 8 in English and 13 in Chinese) included in this study. A flow chart of study selection is shown in Figure 1. We identified 17 and 6 studies that reported on the distribution of number of male sexual partners of MSM in the past 6 and 12 
months, respectively, during 2000-2010 (Table 1). There was no statistical association between the estimated mean/median number of sexual partners and calendar year of the studies (Spearman, $r=-0.13, \mathrm{P}=0.58$ and $r=-$ $0.12, \mathrm{P}=0.63$ for mean/median numbers in past 6 months; $r=-0.26, \mathrm{P}=0.57$ and $r=-0.11, \mathrm{P}=0.84$ for mean/median number in past 12 months, respectively). As no temporal trends of number of partnerships were observed, data from all studies were pooled for subsequent analysis.

According to Figure 2, the overall median number of male sexual partners of Chinese MSM in the past 6 months was estimated to be 1.5 (95\% CI, 1.1-1.8); approximately 7.8\% (95\% CI, 5.3-11.3\%) of Chinese MSM reported having more than 10 sexual partners in the past 6 months. The mean average number of sexual partners for MSM in China in the past 6 months was estimated to be 3.7 (95\% CI, 3.1-
4.3). For studies that reported numbers of partners over the last 12 months, half of MSM stated that they had more than 3.8 (95\% CI 1.5-6.9) sexual partners and $28 \%$ (95\% CI, 16-40\%) had more than 10 sexual partners. The mean number of male sexual partnerships for MSM in China in the last 12 months was found to be 13 (95\% CI, 8-19). Out of 21 studies, only two reported a distribution of number of sexual partners according to different types of part-
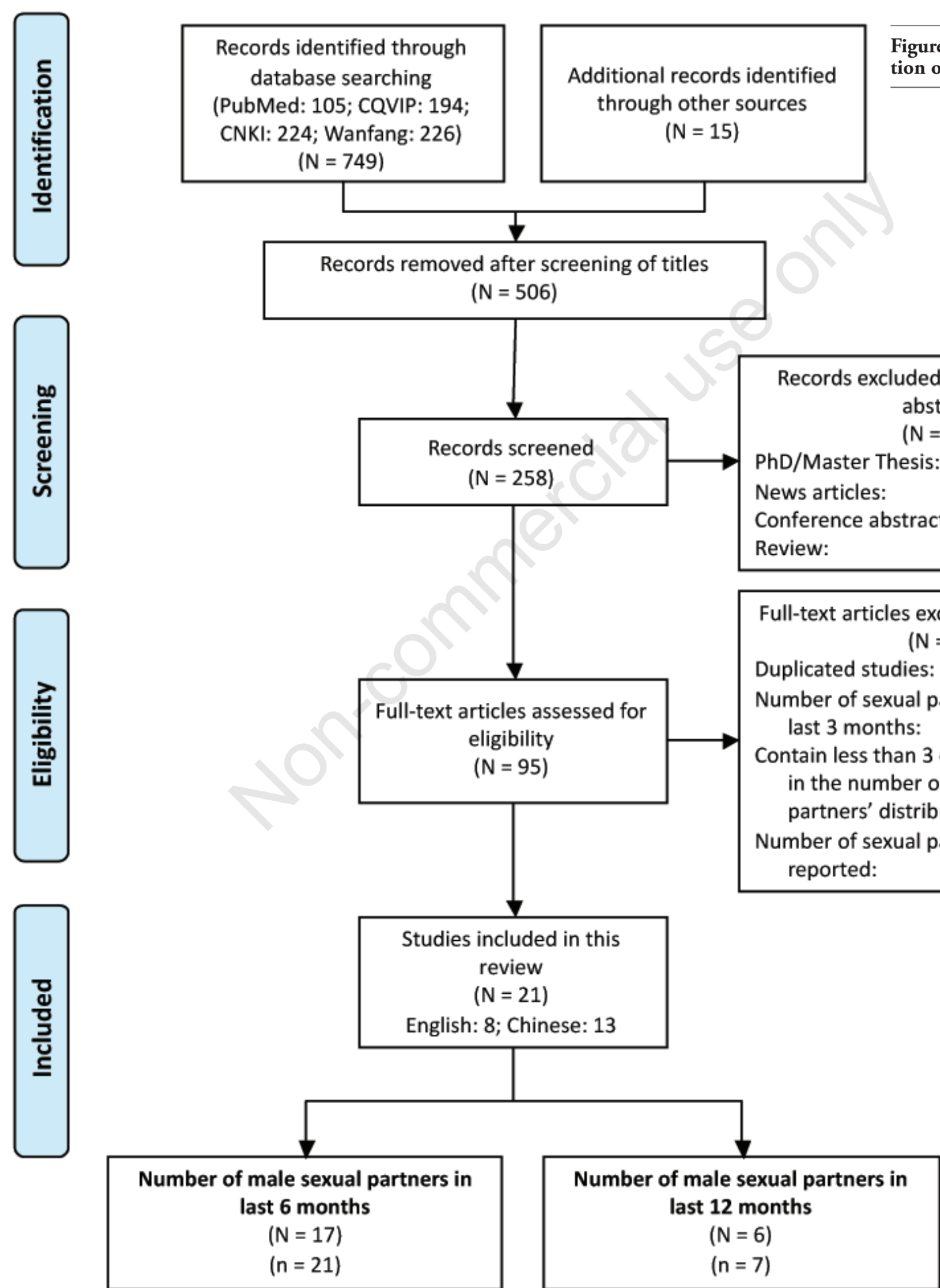

Figure 1. Flow chart for selection of studies. 
nerships (Table 1). ${ }^{8,9}$ Based on these data, we estimate that $31 \%$ of sexual partners of MSM in China are regular partners, $54 \%$ are casual partners, and $16 \%$ are commercial partners.

\section{Discussion}

MSM in China have substantially fewer sexual partners than reported in numerous developed countries. Studies have reported that 37 42\% MSM in Australia, USA and Western European countries have more than 10 sexual partners in the past 6 months, ${ }^{10-16}$ approximately 5 -fold greater than in China. The number of partners reported by MSM in China is also 1.5-4 times fewer than reported in other developing countries such as Brazil and India. ${ }^{17-19}$ Only reports from countries in Africa, ${ }^{20,21}$ Eastern Europe ${ }^{22}$ and neighbouring Asian countries that are traditionally influenced by Chinese culture ${ }^{23-25}$ suggest comparable numbers of sexual partnerships among MSM. This could be due to similar conservative social structures or a tendency to underreport by study participants.

Approximately one-in-three sexual partnerships among MSM in China is a regular partnership, and only approximately $16 \%$ Chinese MSM have engaged in commercial sex in the past 6 months. These data suggest that most Chinese MSM are relatively conservative with regards to sexual mixing and the majority of male sexual intercourse for Chinese MSM takes place in regular partnerships. However only $19.9 \%$ of MSM had consistent condom use with their regular male sexual partners in last six months, which is the lowest condom use rate in comparison with commercial partners (58.0\%) and non-commercial casual partners $(60.4 \%){ }^{26}$

Several limitations should be noted. First, 19 out of 21 studies reported the overall numbers of male sexual partners without specific information about types of sexual relationships. The proportion of MSM who had sex with regular, commercial and non-commercial male sex partners was estimated based on two studies only. ${ }^{89}$ Second, 15 out of the 21 selected studies recruited MSM from MSM venues, (a) CDF of male sexual partners in the last 6 months

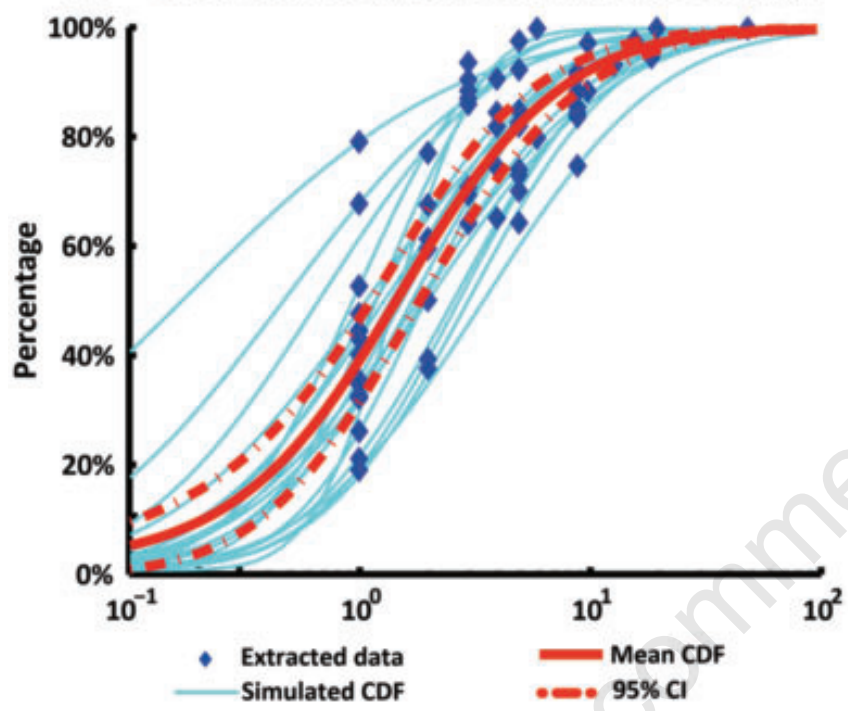

(c) CDF of male sexual partners in the last 12 months

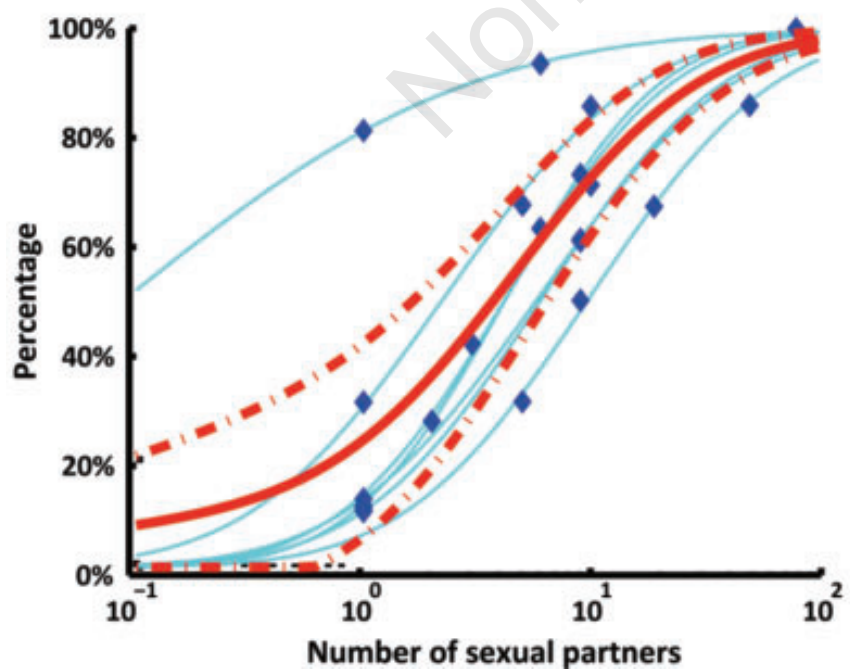

(b) PDF of male sexual partners in the last 6 months

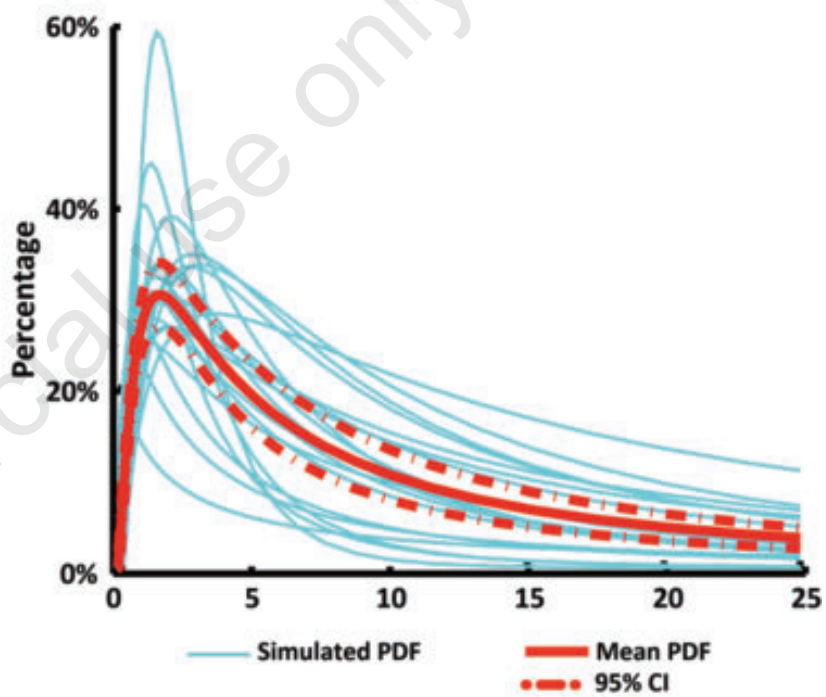

(d) PDF of male sexual partners in the last 12 months

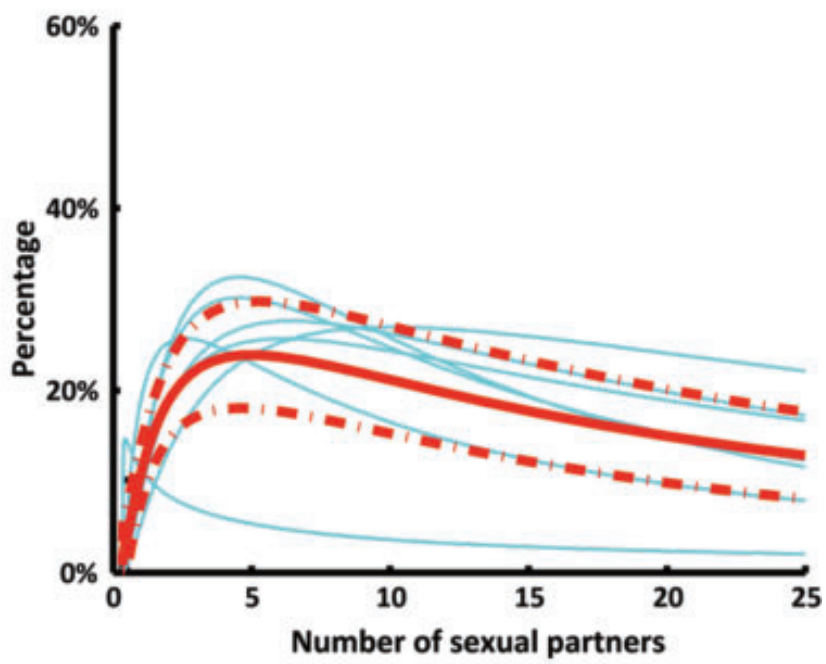

Figure 2. Cumulative distribution function and the deduced probability density function of number of male sexual partners of MSM in China in the past 6 and 12 months. 


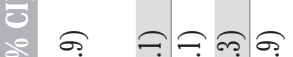

है

=

ป⿻

ช

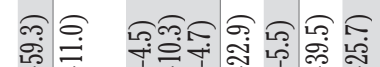

巳ै ê

仓ें

:

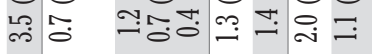

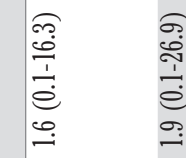

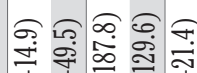

ᄀ่ 才雨

e $e \stackrel{e}{e} e$

की

雨

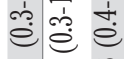

ๆ

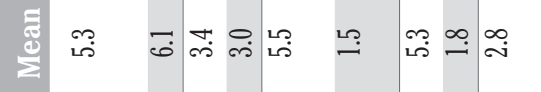

மூ:

के

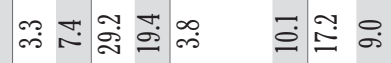

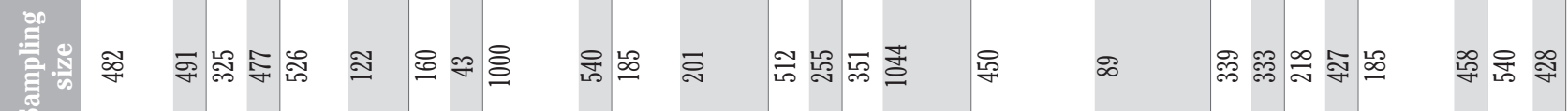

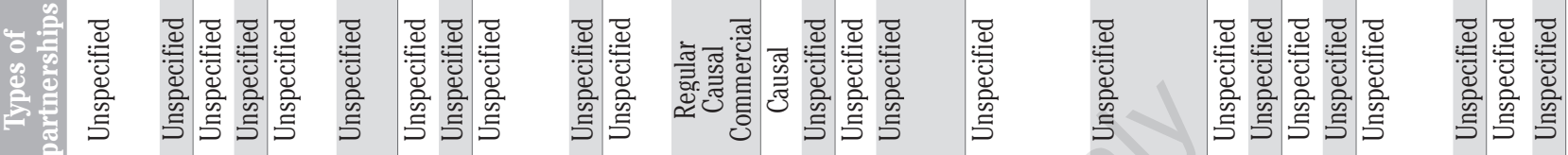

它

ב

总

ชู

要

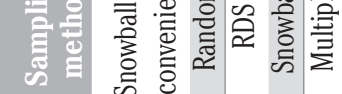

留

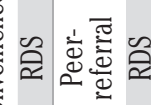

㕆

¿্.

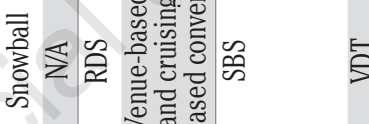

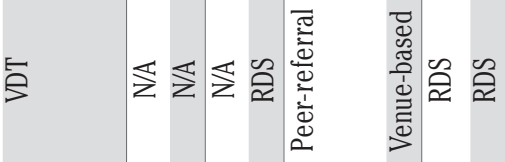

$\pm$

.5

咅

홀

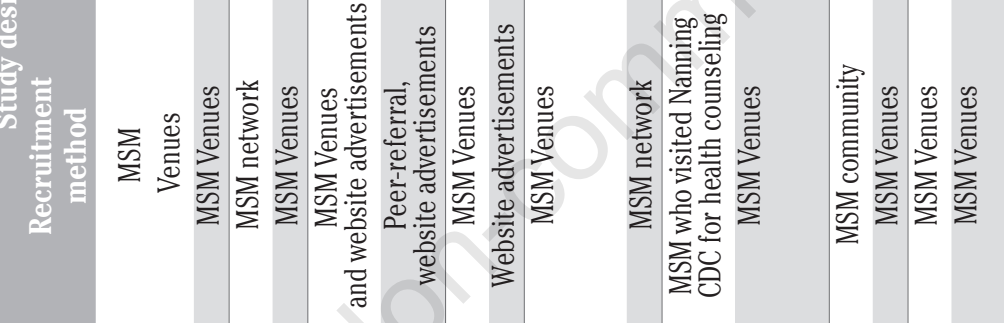

竞

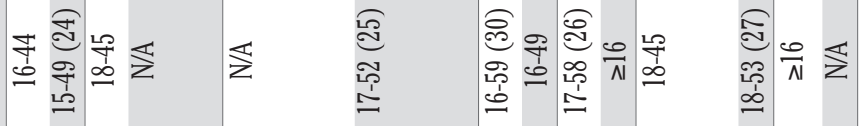

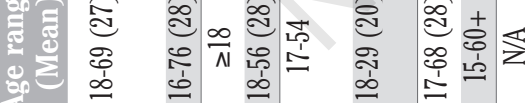

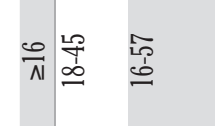

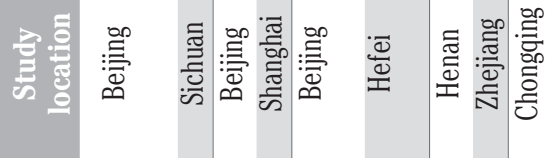

:

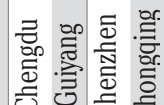

$\overline{\bar{d}}$

节 - or

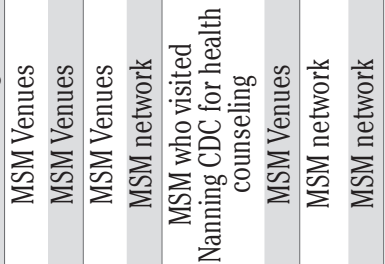

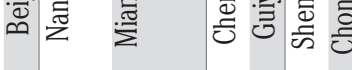

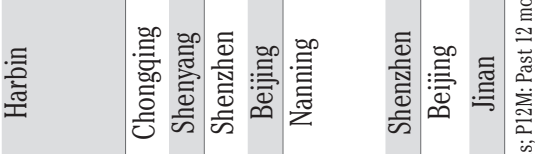

플

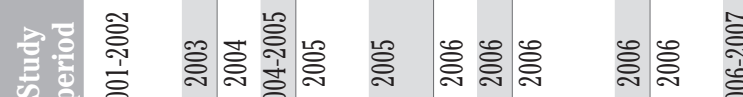

高
高

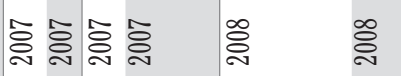

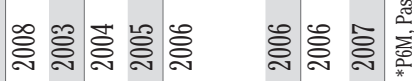

:

के

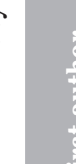
要

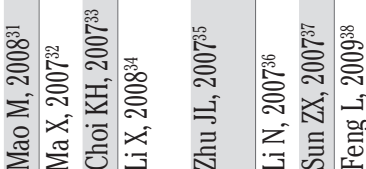

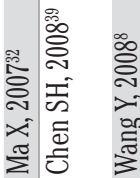

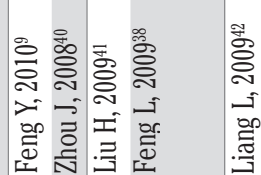

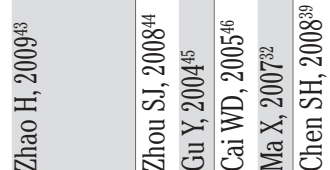

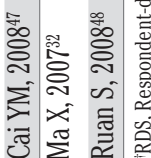


which may potentially participate in higher risk sexual activities ${ }^{27}$ in comparison with MSM recruited via other sampling methods. This may not be representative of the sexual behaviours of the overall MSM population and further investigation into the differences in number of sexual partners due to different sampling methods is required. Third, all the studies were obtained from surveys of selfreported sexual behaviours. Reporting bias, survey consistency, reliability and validity are possible factors contributing to variations across these studies. Fourth, all collated studies reported the number of male sexual partners in major urban cities of China but not in rural regions. However, MSM from rural background are likely to have different education levels, perspectives regarding sexual orientations and sexual behaviors.

The consistently relatively low number of sexual partnerships may not be sufficient to explain the rapid increase of HIV prevalence among MSM in China. Other high-risk sexual behaviours, such as low condom use, ${ }^{28}$ practice of group sex and low testing coverage ${ }^{29}$ may all contribute to the rapid transmission of HIV and other sexually transmissible infections among Chinese MSM. Scaling up prevention efforts should focus on health education about HIV/AIDS and sexually transmissible infections as well as condom distribution and regular HIV/STI testing to curb the further spread of these infections among MSM in China.

\section{References}

1. Wong FY, Huang ZJ, Wang W, et al. STIs and HIV among men having sex with men in China: a ticking time bomb? AIDS Educ Prev 2009;21:430-46.

2. UNGASS. China 2010 UNGASS Country Progress Report (2008-2009). Ministry of Health of the People's Republic of China; 2010.

3. Chow EPF, Wilson DP, Zhang J, et al. Human Immunodeficiency Virus Prevalence Is Increasing Among Men Who Have Sex With Men in China: Findings From a Review and Meta-Analysis. Sexually Transmitted Diseases 2011. [Epub ahead of print]

4. Buchbinder SP, Vittinghoff E, Heagerty PJ, et al. Sexual risk, nitrite inhalant use, and lack of circumcision associated with HIV seroconversion in men who have sex with men in the United States. J Acquir Immune Defic Syndr 2005;39:82-9.

5. Wang N, Wang L, Wu Z, et al. Estimating the number of people living with HIV/AIDS in China: 2003-09. Int $\mathrm{J}$ Epidemiol 2010; 39:ii21-8.

6. Jia MH, Sullivan SG, Lu L, Wu ZY. Curbing the Rapid Spread of HIV Among MSM in China. Jaids-J Acq Imm Def 2010;55:E33-4.

7. Kault D. The shape of the distribution of the number of sexual partners. Stat Med 1996;15:221-30.

8. Wang Y, Zhang HB, Zhang GG, et al. [Effectiveness of AIDS Related Health Education and Behavioral Intervention in Mianyang MSM Group]. J Prev Med Inf 2008;24:962-7.

9. Feng Y, Wu Z, Detels R, et al. HIV/STD prevalence among men who have sex with men in Chengdu, China and associated risk factors for HIV infection. J Acquir Immune Defic Syndr 2010;53:S74-80.

10. Koblin BA, Husnik MJ, Colfax G, et al. Risk factors for HIV infection among men who have sex with men. AIDS 2006;20:731-9.

11. Koblin BA, Chesney MA, Husnik MJ, et al. High-risk behaviors among men who have sex with men in 6 US cities: baseline data from the EXPLORE Study. Am J Public Health 2003;93:926-32.

12. Dukers NH, Renwick N, Prins M, et al. Risk factors for human herpesvirus 8 seropositivity and seroconversion in a cohort of homosexual men. Am J Epidemiol 2000; 151:213-24.

13. O'Brien TR, Kedes D, Ganem D, et al. Evidence for concurrent epidemics of human herpesvirus 8 and human immunodeficiency virus type 1 in US homosexual men: rates, risk factors, and relationship to Kaposi's sarcoma. J Infect Dis 1999;180:1010-7.

14. Fogarty A, Mao L, Zablotska I, et al. The Health in Men and Positive Health cohorts: A comparison of trends in the health and sexual behaviour of HIV-negative and HIVpositive gay men, 2002-2005, National Centre in HIV Social Research Annual Report of Trends in Behaviour. Sydney: University of New South Wales; 2006.

15. Adam PC, Teva I, de Wit JB. Balancing risk and pleasure: sexual self-control as a moderator of the influence of sexual desires on sexual risk-taking in men who have sex with men. Sex Transm Infect 2008;84:4637.

16. Bissessor M, Fairley CK, De Guingand D, et al. Delay in the diagnosis of early syphilis among men who have sex with men: need for greater community and health provider education. Int J Std AIDS 2009;20:52-3.

17. Kumta S, Lurie M, Weitzen S, et al. Bisexuality, sexual risk taking, and HIV prevalence among men who have sex with men accessing voluntary counseling and testing services in Mumbai, India. J Acquir Immune Defic Syndr 2010;53:227-33.

18. Dandona L, Dandona R, Gutierrez JP, et al. Sex behaviour of men who have sex with men and risk of HIV in Andhra Pradesh,
India. AIDS 2005;19:611-9.

19. Kerr-Pontes LR, Gondim R, Mota RS, et al. Self-reported sexual behaviour and HIV risk taking among men who have sex with men in Fortaleza, Brazil. AIDS 1999;13: 709-17.

20. Baral S, Trapence G, Motimedi F, et al. HIV prevalence, risks for HIV infection, and human rights among men who have sex with men (MSM) in Malawi, Namibia, and Botswana. PLoS One 2009;4:e4997.

21. Henry E, Marcellin F, Yomb Y, et al. Factors associated with unprotected anal intercourse among men who have sex with men in Douala, Cameroon. Sex Transm Infect 2010;6:136-140.

22. Stulhofer A, Bacak V, Bozicevic I, Begovac J. HIV-related sexual risk taking among HIV-negative men who have sex with men in Zagreb, Croatia. AIDS Behav 2008; 12:505-12.

23. Hidaka Y, Ichikawa S, Koyano J, et al. Substance use and sexual behaviours of Japanese men who have sex with men: a nationwide internet survey conducted in Japan. BMC Public Health 2006;6:239.

24. Colby D, Cao NH, Doussantousse S. Men who have sex with men and HIV in Vietnam: a review. AIDS Educ Prev 2004;16:45-54.

25. Colby DJ. HIV knowledge and risk factors among men who have sex with men in Ho Chi Minh City, Vietnam. J Acquir Immune Defic Syndr 2003;32:80-5.

26. Chow EPF, Wilson DP, Zhang L. Patterns of condom use among men who have sex with men in China: a systematic review and meta-analysis. AIDS Behav 2011. [Epub ahead of print]

27. Hong FC, Zhou H, Cai YM, et al. Prevalence of syphilis and HIV infections among men who have sex with men from different settings in Shenzhen, China: implications for HIV/STD surveillance. Sex Transm Infect 2009;85:42-4.

28. Zhang B, Li X, Chu Q, et al. [A survey of HIV/AIDS related behaviors among 2250 MSM in nine major cities of China]. Chin J AIDS STD 2008;14:541-7.

29. Chow EPF, Wilson DP, Zhang L. The next era of HIV in China: rapidly spreading epidemics among men who have sex with men. J Acquir Immune Defic Syndr 2010 ;55:e32-3; author reply e33-4.

30. Choi KH, Gibson DR, Han L, Guo Y. High levels of unprotected sex with men and women among men who have sex with men: a potential bridge of HIV transmission in Beijing, China. AIDS Educ Prev 2004;16:19-30.

31. Mao M, Lan YJ, Zhou DL, et al. [Frequency of Condom Use in Anal Copulation Among Homosexual Men]. J Prev Med Inf 2008;24:493-5. 
32. Ma X, Zhang $Q$, He $X$, et al. Trends in Prevalence of HIV, Syphilis, Hepatitis C, Hepatitis B, and Sexual Risk Behavior Among Men Who Have Sex With Men. Results of 3 consecutive respondent-driven sampling surveys in Beijing, 2004 through 2006. J Acquir Immune Defic Syndr 2007;45:581-7.

33. Choi KH, Ning Z, Gregorich SE, Pan QC. The influence of social and sexual networks in the spread of HIV and syphilis among men who have sex with men in Shanghai, China. J Acquir Immune Defic Syndr 2007;45:77-84.

34. Li X, Shi W, Li D, et al. Predictors of unprotected sex among men who have sex with men in Beijing, China. Southeast Asian J Trop Med Public Health 2008;39:99-108.

35. Zhu JL, Zhang HB, Wu HH. [High risk sexual behavior and HIV/STD infection rate among 122 MSM from students]. Chinese Journal of AIDS \& STD 2007;13:350-2.

36. Li N, Wang Z, Sun GQ, Sun DY. [Analysis of HIV/AIDS sentinel surveillance among high risk population in Henan province in 2006]. Chinese Journal of AIDS \& STD 2007;13:427-9.

37. Sun ZX, Lin SF, Wen MQ. [Investigation on
STD and AIDS prevalence for men who have sex with men] Modern Preventive Medicine 2007;34:4130-2.

38. Feng L, Ding X, Lu R, et al. High HIV prevalence detected in 2006 and 2007 among men who have sex with men in China's largest municipality: an alarming epidemic in Chongqing, China. J Acquir Immune Defic Syndr 2009;52:79-85.

39. Chen SH, Zhou J, Zhu JJ, Li KF. [Analysis On Ethological Characters Of MSM In Nanning]. Modern Preventive Medicine 2008;35:902-4.

40. Zhou J, Zhu JJ, Bin H, et al. [A survey of HIV/STD,HBV and HCV infections and risk behaviors among MSM in two central districts of Guiyang city]. Chinese Journal of AIDS \& STD 2008;14:47-8.

41. Liu H, Cai Y, Rhodes AG, Hong F. Money boys, HIV risks, and the associations between norms and safer sex: a respondent-driven sampling study in Shenzhen, China. AIDS Behav 2009;13:652-62.

42. Liang L, Chen ZQ, Miao XF, et al. [Investigation on AIDS knowledge and behaviors among men who have sex with men]. Hebei Medical Journal 2009;31:2654-5.
43. Zhao H, Wang G, Liu H. [The Investigation Report of Male-to-male Contact During the Voluntary Consulation in Daolin District of Harebin]. Guide of China Medicine 2009;7:181-3.

44. Zhou SJ, Pan CB, Meng XR, et al. [The related research on sexual behavior and HIV infection in MSM of Chongqing]. Chin J Infect Control 2008;7:381-4.

45. Gu Y, Qu P, Xu L, et al. [Survey of knowledge, attitude, behavior and practice related to STI/HIV among male homosexuality in Shenyang]. Chin J Public Health 2004;20:573-4.

46. Cai WD, Fen TJ, Tan JQ, et al. [A Survery Of The Characteristics And STD/HIV Infection Of Homosexuality In Shenzhen]. Modern Preventive Medicine 2005;32:32830.

47. Cai YM, Liu H, Pan P, et al. [High risk behaviors among 458 men who have sex with men in Shenzhen]. South China J Prev Med 2008;34:13-5.

48. Ruan S, Yang H, Zhu Y, et al. HIV prevalence and correlates of unprotected anal intercourse among men who have sex with men, Jinan, China. AIDS Behav 2008; 512:469-75. 